\title{
42. LOS GÉNEROS HYPOCHAERIS L. Y ACHYROPHORUS VAILL. (COMPOSITAE, CICHORIEAE): NUEVOS TAXONES Y COMBINACIONES
}

\author{
Salvador TALAVERA, María de los Ángeles ORTIZ, Francisco Javier JIMÉNEZ, \\ Karin TREMETSBERGER y María TALAVERA
}

Recibido el 21 de octubre de 2015, aceptado para su publicación el 15 de noviembre de 2015

The genus Hypochaeris L. and Achyrophorus Vill.: new taxa and new combinations

Palabras clave. Corología, sistemática, tipificación, Región Mediterránea.

Key words. Chorology, systematic, typification, Mediterranean basin.

Estudios de filogenia molecular llevados a cabo en el género Hypochaeris sensu DeFilipps (1976) han puesto de manifiesto su carácter parafilético (Enke et al., 2012; Tremetsberger et al., 2013), y la existencia de seis clados con fuerte respaldo, que se corresponden con otros tantos géneros, muchos de ellos reconocidos desde la primera mitad del siglo XIX (Candolle, 1838).

En lo concerniente al territorio de "Flora iberica" se han reconocido tres géneros. Trommsdorffia Bernh, Syst. Verz.: 102 (1800), un género eurasiático con 3 especies reconocidas, de las que solo $T$. maculata (L.) Vernh., Syst. Verz.: 140 (1800); [三 Hypochaeris maculata L., Sp. Pl.: 810 (1753), basión.; = Hypochaeris pyrenaica Senn. in Sennen, P1 d' Espagne 1922: No 4443 (1822), in sched.] alcanza el norte de España [And. Esp.: B Bu $\mathrm{Ge} \mathrm{Hu} \mathrm{L} \mathrm{Le} \mathrm{Na} \mathrm{S} \mathrm{So].} \mathrm{Este} \mathrm{género} \mathrm{se} \mathrm{diferencia}$ de los demás géneros que han sido escindidos de Hypochaeris, por el involucro densamente viloso, lígulas con el tubo viloso y los vilanos formados por 20-26 pelos plumosos, no dilatados en la base, dispuestos en una sola fila. En los otros dos géneros con representación en la Península Ibérica y Baleares, Hypochaeris
L. y Achyrophorus Vaill., la revision efectuada hace necesarias algunas combinaciones nomenclaturales y la descripción de algunos táxones nuevos, resultados que constituyen el principal objetivo del presente trabajo.

Hypochaeris L., Sp. P1.: 810 (1753)

$\equiv$ Porcellites Cass. in Cuvier, Dict. Sci.

Nat. 43: 42 (1826)

$\equiv$ Hypochaeris sect. Porcellites (Cass.) DC., Prodr. 7: 91 (1838)

Especie tipo: Hypochaeris radicata L. Hypochaeris sect. Arachnites DC., Prodr. 7: 90 (1838)

Especie tipo: Hypochaeris arachnoides Poir.

= Hypochaeris sect. Diplostephanae Bisch., Beitr. Fl. Deustchl.: 139 (1851)

Especie tipo: Hypochaeris glabra L.

Hierbas anuales, o perennes, rizomatosas y a veces también estoloníferas, caulescentes, por lo general escaposas, erectas o ascendentes, unio pluricaules, glabras o con pelos unicelulares blancos en las hojas y en la base del tallo, inermes; raíz vertical, delgada $\mathrm{o} \pm$ napiforme, $\mathrm{o}$ bien rizoma premorso con raíces tuberosas que

\footnotetext{
Este trabajo ha sido financiado por el proyecto Flora iberica (CGL2012-32914) y cofinanciado con fondos FEDER
} 
se originan en el cuello radical, a veces también con estolones \pm desarrollados. Tallos cilíndricos, estriados, por lo general escapiformes, rara vez foliosos, con una sola hoja bien desarrollada, glabros o \pm vilosos en la mitad inferior, ramificados en la mitad superior, en pocas ocasiones simples. Hojas lineares, linearelípticas u oblanceoladas, enteras, dentadas, lobadas o pinnatífidas, a veces pectinadas o runcinadas, glabras o más frecuentemente pelosas por ambas caras; las basales formando una roseta, pecioladas, con el pecíolo ancho y poco diferenciado; las caulinares, cuando existen, de morfología similar a las basales pero más pequeñas. Capítulos erectos antes de la antesis, por lo general agrupados en una inflorescencia cimosa y oligocéfala, largamente pedunculados, rara vez solitarios, multifloros; pedúnculos cilíndricos, a veces ensanchados en el ápice en la fructificación, glabros, con varias brácteas. Involucro cilíndrico en la floración y fructificación, a veces subcónico en la fructificación, con 4-5 series de brácteas adpresas crecientes en tamaño hacia el interior del capítulo; brácteas herbáceas, verdosas, glabras por ambas caras, ciliadas en el ápice, poco acrescentes en la fructificación, con una quilla dorsal bien marcada y a veces cubierta de setas aculeiformes. Receptáculo convexo en la dispersión, alveolado, paleáceo, con las páleas interflorales linear-subuladas, membranáceas, con el nervio medio bien diferenciado, alcanzando la altura del vilano en la fructificación, glabro. Flores liguladas, con la lígula culminada por 5 dientes, hermafroditas, las externas del capítulo algo mayores que las internas. Corola vilosa en la base del limbo, amarilla, las más externas del capítulo a menudo con el dorso rosado, verdoso o purpúreo, con los dientes de la lígula provistos de un mucrón subapical \pm desarrollado, sobre todo los laterales. Anteras amarillas. Ramas estilares amarillas. Aquenios homomorfos, con o sin pico, o con más frecuencia dimorfos, los externos del capítulo sin pico y los centrales con pico, con 14 ó 16 costillas longitudinales cubiertas de espículos, los carentes de pico \pm obcónicos y los que lo tienen \pm fusiformes, atenuados hacia los extremos del cuerpo o parte seminífera, pardo-rojizos, con los espacios intercostales blanco-azulados, todos con vilano. Vilano plumoso, blanco, persistente, formado por 26-50 pelos y escamas setáceas dispuestos en 2 o más filas, soldados entre sí en la base formando una corona fuertemente unida al aquenio; la fila más externa por lo general integrada por pelos escábridos, algo más pequeños que las filas más internas; las más internas por escamas plumosas, y en la fila más interna de los aquenios sin pico por escamas densamente vilosas en el tercio inferior del vientre. $x=4,5$.

\section{Observaciones}

Género monofilético que integran 4 especies distribuidas por la Región Mediterránea, aunque $H$. radicata y $H$. glabra se han introducido en gran parte de las regiones húmedas del Mundo. De ellas, H. glabra, es autocompatible y autógama (Ortiz et al., 2006), y posee $2 n=10$ cromosomas (Tremetsberger et al., 2004), mientras que las otras tres ( $H$. radicata, $H$. salzmanniana y $H$. arachnoides) son autoincompatibles y tienen $2 n=8$ cromosomas (Oberprieler \& Vogt, 2002; Tremetsberger et al., 2004), aunque en $H$. salzmanniana algunas de las poblaciones españolas son autocompatibles y autógamas o semi-incompatibles (ver observaciones de esta especie). Estudios moleculares de secuenciación han mostrado que $H$. glabra es la especie basal del género y $H$. radicata es la hermana filogenética de $H$. salzmanniana y $H$. arachnoides (Tremetsberger et al., 2005). Este resultado es coincidente con los de estudios filogeográficos llevados a cabo con AFLP (Ortiz et al., 2009), que han mostrado además que la mayor diversidad genética intrapoblacional en las cuatro especies se encuentra en el ecotono del valle del río Sebou con el Atlas Medio 
(Marruecos), donde probablemente se originó el género Hypochaeris.

En el NW de África (Marruecos, Argelia y Túnez) vive además $H$. arachnoides Poir. in Lam., Encycl. 5: 572 (1804), una especie anual, parecida a $H$. salzmanniana en la morfología y en el número cromosómico $(2 n=8)$, pero con los pedúnculos cilíndricos en la fructificación y una valencia ecológica mucho más amplia que la de $H$. salzmanniana.

\section{Clave para las especies}

1. Corola de las flores más externas del capítulo de (4)6,5-10 mm, que apenas sobresale del involucro; anteras $(1,3) 1,5-2 \mathrm{~mm}$; hierba anual, por lo general glabra .................... 3. H. glabra

- Corola de las flores más externas del capítulo de (8) 10-20 mm, que sobresale bastante del involucro; anteras (3)3,5-5 mm; hierba anual o perenne, por lo general pelosa, al menos en las hojas ...... 2

2. Hierba perenne, con raíces \pm fusiformes, fasciculadas o divaricadas, a veces estolonífera; pedúnculo cilíndrico en la fructificación; capítulos con aquenios homomorfos y con pico, o bien dimorfos, los más externos sin pico y los internos con pico; vilano (6)7-11(12) mm; aquenios con 14 costillas longitudinales .........

1. H. radicata

- Hierba anual, con una sola raíz, axonomorfa, sin raíces fusiformes; pedúnculo con el ápice fuertemente ensanchado en la fructificación; capítulo con aquenios dimorfos, los externos sin pico y los internos con pico; vilano 10-16 mm; aquenios con 16 costillas longitudinales

2. H. salzmanniana

\section{Hypochaeris radicata L., Sp. Pl.: 811 (1753)}

$\equiv$ Achyrophorus radicatus (L.) Scop., Fl. Carniol. ed. 2, 2: 117 (1772)

$\equiv$ Porcellites radicata (L.) Cass. in Cuvier, Dict. Sci. Nat. 43: 43 (1826)

Ind. loc.: "Habitat in Europae cultioris pascuis" [Lectotipo: designado por Scott in Jarvis et al. (1993: 109): LINN No. 959.5]

\section{Ecología, fenología y distribución}

En sotobosque de alcornocales, quejigares, encinares, pinares, acebuchares, etc., o en sus matorrales de sustitución -brezales, jarales, aulagares, etc.- de ambiente húmedo, a veces también en prados, taludes, márgenes de lagunas e incluso en arenales costeros; 0-2000 m. (IV) V-VII(X). Región Mediterránea; naturalizada en casi toda Europa, Asia, América, Sudáfrica y Australia. Toda la Península Ibérica. And. Esp.: A Ab Al Av B Ba Bi Bu C Ca Cc Co CR Cs Cu Ge Gr Gu H Hu J L Le Lo Lu M Ma Mu $\mathrm{Na} O$ Or P PM[Ib Mll Mn] Po S Sa Se Sg So SS T Te To V Va Vi Z Za. Port.: AAl Ag BA BAl BB BL DL E Mi R TM

\section{Observaciones}

Especie autoincompatible, aunque muchas de sus poblaciones presentan plantas parcial o totalmente autocompatibles (Ortiz et al., 2006). Se comporta como una buena invasora, $y$ estudios filogeográficos recientes han puesto de manifiesto que comprende varias razas genéticas y morfológicamente bien diferenciadas (Ortiz et al., 2007 \& 2009), que se reconocen aquí para el territorio como subespecies: subsp. radicata, subsp. platylepis (Boiss.) Jahand. \& Maire y subsp. rocinensis, ésta última descrita en este trabajo. Además de las mencionadas, se conoce la subsp. neapolitana (DC.) Nyman, Consp. Fl. Eur.: 470 (1879) [三H. neapolitana DC., Prodr. 7: 91 (1838), basión.; =H. radicata var. heterocarpa Moris, Fl. Sardoa 2: 487 (1840-1843); 三H. radicata subsp. heterocarpa (Moris) Arcang., Consp. Fl. Ital.: 414 (1882)], del C y E de la Región Mediterránea, y que se caracteriza por presentar el rizoma fuertemente leñoso en el ápice, sus brácteas externas del involucro anchas, envés de las lígulas externas del capítulo purpúreo y los aquenios dimorfos, los externos del capítulo sin pico y los centrales con pico.

\section{Clave para las subespecies}

1. Brácteas más externas del involucro de $(2,1) 2,5-4,5 \mathrm{~mm}$ de anchura, ovadas, \pm cordiformes, con el margen \pm ondulado y blanco; hojas por lo general pectinadas, con los lóbulos obtusos; capítulos con los aquenios casi siempre dimorfos, rara vez 
homomorfos

b. subsp. platylepis

- Brácteas más externas del involucro de 0,7-1,8 $\mathrm{mm}$ de anchura, \pm lanceoladas o elípticas, con el margen plano y verdoso, rara vez ondulado y blanquecino; hojas enteras, dentadas, lobadas o pinnatífidas, con frecuencia runcinadas; capítulos con los aquenios homomorfos, rara vez dimorfos

2

2. Planta sin estolones; hojas por lo general oblanceoladas, lobadas o pinnatífidas, con frecuencia runcinadas, rara vez dentadas, por lo general muy pelosas, con pelos de $(0,5) \quad 1,5-3,5 \mathrm{~mm}$ en su haz; capítulos con los aquenios homomorfos, rara vez dimorfos ............................ a. subsp. radicata

- Planta estolonífera; hojas lineares o estrechamente oblanceoladas, enteras o débilmente dentadas, glabras o glabrescentes, con pelos de 0,5-1 mm por su haz; capítulos con los aquenios homomorfos

c. subsp. rocinensis

a. subsp. radicata

$=H$. salina Gren. in Mém. Soc. Emul. Doubs., ser. 3, 10: 456 (1869)

$=H$. gracilis Sennen in Sennen, P1. d' Espagne 1926: No. 5788 (1926), in sched. $=H$. radicata subsp. ericetorum Soest in Ned. Kruidk. Arch. 57: 242 (1950)

\section{Ecología, fenología y distribución}

Prados de siega, sotobosque de alcornocales, pinares, rebollares, etc.; 0-2000 m. IV-VIII(X). Región Mediterránea; naturalizada en casi toda Eurasia, América, Sudáfrica y Australia. Casi toda la Península Ibérica, desde Sierra Morena y la Sierra de Cazorla hasta el norte de España, y falta en gran parte de las sierras Béticas y Penibéticas. And. Esp.: A Ab Al Av B Ba Bi $\mathrm{Bu} \mathrm{C}$ Cc Co CR Cs Cu Ge Gr Gu H Hu J L Le Lo Lu M Mu Na O Or Po S Sa Se Sg So SS T Te To V Va Vi Z Za. Port.: AAl Ag BA BAl BB BL DL E Mi R TM.

\section{Observaciones}

En el oeste de la Península Ibérica, sobre todo en Sierra Morena, es frecuente que plantas con aquenios homomorfos convivan con otras de aquenios dimorfos -los externos del capítulo sin pico y los centrales con pico-, pero ambos tipos de plantas son interfértiles y genéticamente idénticas.

b. subsp. platylepis (Boiss.) Jahand. \& Maire, Cat. P1. Maroc: 831 (1934)

$\equiv H$. platylepis Boiss., Voy. Bot. Espagne: 376 (1841) [basión.]

Ind. loc.: "In siccis et ad margines viarum regionis calidae superioris et montanae, rupestris circà Alhaurin ad radices montis Sierra de Mijas (forma fol. invol. que pilosis), circà Coin, Estepona, Sierra Nevada pars inferior circà San Gerónimo. Alt. 1500'-5000'. Fl. Jun. Jul.”.

Lectotipo: designado por Burdet et al. (1983:786): G.

\section{Ecología, fenología y distribución}

Sotobosque de alcornocales, quejigares, encinares, acebuchares, etc., en substratos por general arcillosos; 10-1300 m. (IV)V-VII. Sur de España y NW de África (Argelia y Marruecos). Valle del Guadalquivir y Sistema Bético occidental, raro en la Penibética. Esp.: $\mathrm{Al}$ Ca Co Gr J Ma Se.

\section{Observaciones}

En Sierra Nevada [Esp.: Al Gr] y en las Sierras Subbéticas [Esp.: J Se] son frecuentes plantas con los aquenios homomorfos, todos con el pico bien desarrollado, pero con las brácteas externas del involucro y la morfología de las hojas semejantes a las plantas de la serranía de Ronda y sierra del Aljibe, donde abundan más las que presentan los aquenios dimorfos. En la Península Tingitana las brácteas externas del involucro son anchas como las del sur de España, pero en las montañas del Rif y sobre todo en el Atlas las brácteas externas son más estrechas, parecidas a las de $H$. radicata subsp. radicata, pero los aquenios de los capítulos son por lo general dimorfos. No obstante, estudios moleculares han demostrado que la distancia genética entre las poblaciones 
del Atlas marroquí y las andaluzas es muy pequeña, y no se cree oportuno reconocer las poblaciones del Atlas como un taxón propio.

c. subsp. rocinensis M. Á. Ortiz \& Talavera, subsp. nov.

Holotype: ESPAÑA. Huelva. Almonte. El Acebrón. La Rocina. Humedales en el alcornocal, 27-V-2014. Leg. M. Talavera \& S. Talavera, no. 403/14 (SEV 285032). Isotype: ibídem (SEV 285491).

Paratype: Huelva. Almonte. El Acebrón, alcornocal inundable de La Rocina, 19-VI-2001, S. Talavera, M.Á. Ortiz, R. Berjano \& C. de Vega (SEV 286508); Almonte. Reserva Biológica de Doñana. Laguna de Santa Olalla, 22-VI-1974, B. Cabezudo (SEV 18828); ídem, La Algaida de Doñana, 31-V-1980, S. Castroviejo \& G. López (MA 445967); ídem, Laguna de La Algaida, 7-VI-2002, S. Talavera, M.Á. Ortiz, R. Berjano \& C. de Vega SEV (286509); ídem, Laguna del Corchuelo, 7-VI-2002, S. Talavera, M.Á. Ortiz, R. Berjano \& C. de Vega (SEV 286510).

Stoloniferous herb, contrasting with the lack of stolons in Hypochaeris radicata subsp. radicata; leaves linear or narrowly oblanceolate, entire or weakly dentate, glabrous or glabrescent with hairs $0.5-1 \mathrm{~mm}$ on the surface-contrasting with oblanceolate, lobate or pinnatifid, commonly runcinate, rarely dentate, usually very hairy with hairs (0.5-) 1-3.5 mm on the surface in Hypochaeris radicata subsp. radicata; achenes homomorphic, all with a beak - whereas achenes homomorphic and all beaked, or dimorphic and the most external in the capitulum not beaked, and the innermost beaked, in Hypochaeris radicata subsp. radicata.

Hierba perenne, rizomatosa, estolonífera; rizoma vertical, con raíces tuberificadas y fusiformes en el cuello radical, desde donde emite estolones subterráneos \pm largos que originan varias rosetas de hojas, formando poblaciones clonales, rara vez leñosos cuando muy viejos. Tallos de (35)50-100 cm x 1,1-3 mm de grosor (en la base), escapiformes, glabros o vilosos en la base, con pelos unicelulares de 1-1,5 mm. Hojas 5,5-25(40) x 1,2-2(4) cm, lineares o linear-elípticas, enteras $\mathrm{o} \pm$ dentadas, con los dientes agudos, laxamente seríceas por ambas caras, con pelos unicelulares de 0,5-1,5 mm por su haz, a veces glabras, solo con el margen ciliado. Involucro 9-16(18) x 5,5-6(7) mm, cilíndrico en la floración; brácteas con el dorso glabro, por lo general sin setas aculeiformes en el nervio medio; las más externas 2-4(4,5) x 1,4-1,8 mm, elípticas o lanceoladas, de margen casi siempre plano, a veces algo ondulado y blanquecino; las internas (8)13-16 x 2-2,4 mm, linear-lanceoladas, con margen membranoso estrecho. Corola 15-18 $\mathrm{mm}$, amarilla o las más externas del capítulo con el dorso \pm verdoso. Anteras 4-4,3 mm. Ramas estilares 0,4-0,7 mm. Aquenios 10-16 $\mathrm{mm}$, homomorfos, con pico de 5,5-11 $\mathrm{mm}$. Vilano 8-9,5 $\mathrm{mm}$.

\section{Ecología, fenología y distribución}

Herbazales perilagunares, en substratos higroturbosos; 10-30 m. (V)VI-VII. Endemismo del sur de la Península Ibérica: Parque Natural de Doñana y en el entorno del arroyo de La Rocina. Esp.: H.

2. Hypochaeris salzmanniana DC., Prodr. 7: 91 (1838)

$\equiv$ H. glabra subsp. salzmanniana (DC.) Maire in Jahandiez \& Maire, Cat. P1. Maroc.: 831 (1934).

Ind. loc.: "in agro Tingitano detexit cl. Salzmann. H. dimorpha Salzm.! pl. exs.”.

Lectotipo (designado aquí por S. Talavera): "Hypochaeris dimorpha / 1815 / Salzmann [manuscrito Salzmann] / Hypochaeris salzmanniana [manuscrito A. P. De Candolle]", etiqueta única (G-DC 00458868)]. 
Ecología, fenología y distribución

Herbazales en arenales marítimos; 10-50 m. III-V(VI). Litoral atlántico del NW de Marruecos, y del S de España. Cádiz, desde Chiclana de la Frontera hasta la desembocadura del río Palmones (La Línea de la Concepción). Esp.: Ca.

\section{Observaciones}

Esta especie es muy frecuente en Marruecos, desde la desembocadura del río Sebou (Kenitra) hasta las playas de Tánger. En los bosques de la Mamora la especie presenta su mayor diversidad genética, por lo que se supone que aquí se ubica el centro de origen de la especie. En su migración hacia el norte la especie va perdiendo diversidad, siendo las poblaciones cercanas a Tánger las más pobres de Marruecos. Durante las glaciaciones del Cuaternario la especie cruzó el Estrecho de Gibraltar al menos dos veces, una hacia la Bahía de Algeciras y otra hacia el estuario del río Barbate. Posteriormente, estas últimas poblaciones migraron hacia la ensenada de Tarifa, alrededor de la base de la Sierra de San Bartolomé, donde las poblaciones presentan los parámetros de diversidad genética más bajos de toda la especie (Ortiz et al., 2007).

Todas las poblaciones estudiadas, tanto de Marruecos como de España, son autoincompatibles, excepto las del entorno de la Sierra de San Bartolomé, que son autocompatibles; en las playas de El Palmar (Vejer de la Frontera) las poblaciones son mixtas, y están integradas por plantas autocompatibles y autoincompatibles. Un estudio morfométrico de los capítulos en antesis de las plantas autocompatibles ha mostrado que el diámetro es siempre menor que el de las plantas autoincompatibles (Ortiz et al., 2006) y, como cabría esperar, las anteras de las flores son menores (3-3,5 mm) que las de las plantas autoincompatibles (4-5 mm).

Recientemente, se ha descrito una nueva subespecie de $H$. salzmanniana $[H$. salzmanniana subsp. maroccana Förther \& Podlech in Sendtera 8: 41 (2002)], pero todo el material incluido en esta subespecie corresponde en realidad a otra especie, Hypochaeris arachnoides Poir. in Lam., Encycl. 5: 572 (1804) [- H. grandiflora Sennen \& Mauricio in Sennen, Diagn. Nouv.: 236 (1936), non Ledeb. (1833), nec Phil. (1894); = H. multicaulis Sennen \& Mauricio in Sennen, Diagn. Nouv.: 236 (1936)], que al igual que $H$. salzmanniana es anual y tiene $2 n=8$ cromosomas, y que se extiende por las montañas y valles de casi todo el NW de África (Marruecos, Argelia y Túnez).

3. Hypochaeris glabra L., Sp. P1.: 811 (1753) $\equiv$ H. stellata Gaterau, Descr. P1. Montauban: 140 (1789), nom. illeg.

$\equiv H$. radicata subsp. glabra (L.) Mateo \& Figuerola, Fl. Analit. Prov. Valencia: 369 (1987)

$\equiv H$. arachnites Link, Handbuch 1: 792 (1829), nom. illeg.

$\equiv H$. capensis Less., Syn. Gen. Compos.: 130 (1832), nom. illeg.

Ind. loc.: "Habitat in Dania, Germania, Belgio.".

Lectotipo: designado por Alavi (1983): LINN No. 959.4.

= Scorzonera taraxacifolia Jacq., Icon. P1.

Rar. 1: 16, t. 160 (1781-1786)

$\equiv$ Podospermum taraxacifolium (Jacq.)

Sweet, Hort. Brit.: 207 (1826)

= Hypochaeris hispida Roth in Usteri, Ann.

Bot. 14: 30 (1795)

= Hyoseris tenella Thunb., Prod. Pl. Cap.: 139 (1800)

= Hypochaeris simplex Mérat, Nouv. Fl. Env. Paris: 310 (1812)

= H. pusilla Poir. in Lamarck, Encycl. Suppl. 4: 530 (1816)

=Achyrophorus balbisii Hornem., Hort. Bot. Hafn., Suppl.: 91 (1819) 
$=$ Hypocaaeris contexta Wallr. in Linnaea

14: 663 (1841)

$=H$. candollei Regel in Linnaea 16: 64 (1842)

$=H$. intertexta Peterm. in Flora 27: 481 (1844)

$=H$. pumila Phil. in Anales Univ. Chile, I, Mem. Ci. Lit. 87: 322 (1894)

\section{Ecología, fenología y distribución}

Herbazales de sotobosque y claros de pinares, alcornocales, encinares, etc., o en sus matorrales de sustitución, brezales, jarales, etc., en substratos preferentemente ácidos y de textura arenosa; 10-1604 m. III-V(VI). Región Mediterránea; naturalizada en Eurasia y en otras muchas partes del Mundo. Casi toda la Península Ibérica, aunque es más rara o falta en algunas provincias del norte. Esp.: A Al Av B Ba C Ca Cc Co CR Cu Ge Gr Gu H J Lo M Ma Mu O Or PM[Mll] Sa Se Sg So T Te To V Va Vi Z Za. Port.: AAl Ag BAl BL DL E.

\section{Observaciones}

Es la única especie del género en la que todas las plantas estudiadas son autocompatibles y autógamas (Ortiz et al., 2006), por lo que las flores son pequeñas, y apenas sobresalen del involucro. En el sur de Inglaterra y en el norte de Francia se han descrito híbridos entre $H$. glabra y $H$. radicata. Los híbridos de primera generación tienen $2 n=9$ cromosomas, son perennes y morfológicamente parecidos a $H$. radicata, pero son estériles. Se han obtenido híbridos experimentales de estas dos especies pero siempre cuando $H$. radicata actúa como donador de polen (Parker, 1975).

Como en las otras dos especies del género, las poblaciones del norte de Marruecos son las que presentan mayor diversidad genética (Ortiz et al., 2009), por lo que también se le supone el centro de origen.

En el territorio de Flora iberica, las plantas más comunes son heterocarpas, con los aquenios centrales del capítulo con pico y los externos sin él. Estas plantas se corresponden con $H$. glabra s.s. [ =H. minima Cirillo, Pl. Rar. Neapol. 1: XXIX, tab. 10 (1788) $\equiv H$. glabra subsp. minima (Cirillo) Arcang., Comp. Fl. Ital.: 414 (1882); = H. dimorpha Brot., Fl. Lusit. 1: 332 (1804); =H. adscendens Brot., Phytogeogr. Select. 1: 55, tab. 25 (1816); = H. boscoi Sennen in Sennen, Pl. Espagne 1929: No. 7237 (1929), in sched.]. Mucho más raras aquí son las plantas homocarpas, unas con todos los aquenios del capítulo con pico [ $=H$. balbisii Loisel., Not. Fl. France: 124 (1810); = H. glabra var. rostrata Coss. \& Germ., Fl. Env. Paris: 427 (1845); = H. glabra var. loiseleuriana Godr. in Grenier \& Godron, Fl. France 2: 293 (1850); = H. glabra var. isocarpa Bish., Fl. Deutschl.: 142 (1851)], y otras sin él $[=H$. glabra var. erostris Coss. \& Germ., Fl. Env. Paris: 427 (1845), excluida sinonimia].

Achyrophorus Vaill. in Königl. Akad. Wiss. Paris Phys. Abh. 5: 739 (1754)

三Seriola L., Sp. Pl., ed. 2, 2: 1139 (1763), nom. illeg.

E Hypochaeris sect. Achyrophorus (Vaill.)

W. D. J. Koch., Syn. Fl. Germ. Helv., ed. 2: 491 (1844)

EHypochaeris subg. Achyrophorus (Vaill.) Peterm., Deutschl. F1.: 336 (1846-1849)

Especie tipo: Hypochaeris achyrophorus L., Sp. Pl.: 810 (1753) (Designada por Greuter et al., 2005: 167].

= Agenora D. Don in Edimb. N. Phil. J. 6: 310 (1829)

= Piptopogon Cass. in Cuvier, Dict. Sci. Nat. 48: 434, 507 (1827)

ESeriola subg. Piptopogonopsis Batt. in Bull. Soc. Hist. Nat. Afrique N. 3: 23 (1912)

Sufrútices o hierbas anuales, caulescentes, erectas o ascendentes, uni- o pluricaules, glabrescentes, con un indumento farinoso formado por pelos blancos muy cortos en 
el ápice de los pedúnculos y en la base del involucro, glabras o con mayor frecuencia \pm vilosas en las hojas y, a veces, en la base de los tallos, con pelos unicelulares, blancos, con la base engrosada, inermes, aunque a menudo las brácteas del involucro suelen tener setas aculeiformes muy rígidas, casi espinescentes; sistema radical recto, leñoso y a veces ramificado en el ápice, o herbáceo y delgado. Tallos cilíndricos, estriados, escapiformes o foliosos, glabros o vilosos en la base, simples o ramificados, a veces casi desde la base. Hojas oblanceoladas o subespatuladas, enteras, dentadas, lobadas o subpinnatífidas, con el lóbulo terminal mucho mayor que los laterales, glabras o pelosas por ambas caras; las basales formando rosetas, pecioladas, con el pecíolo estrecho \pm largo o ancho y poco diferenciado del limbo; las caulinares de la morfología de las basales pero más pequeñas. Capítulos erectos o péndulos antes de la antesis, por lo general agrupados en una inflorescencia cimosa policéfala o con más frecuencia oligocéfala, largamente pedunculados, a veces solitarios, multifloros; pedúnculos cilíndricos, con indumento laxo y farinoso, a veces acompañado de setas rígidas \pm aculeiformes en el ápice. Involucro \pm cilíndrico en la floración y fructificación, con 2-3 series de brácteas adpresas, las externas mucho más cortas que las internas; brácteas elípticas o lanceoladas, ciliadas en el ápice, verdosas, con una quilla dorsal generalmente acompañada de 1-3(5) filas de setas espinulosas de base ensanchada. Receptáculo alveolado, paleáceo, con las páleas interflorales linear-subuladas, membranáceas, con el nervio medio bien marcado y que alcanzan la altura del vilano en la fructificación, glabro. Flores liguladas, con la lígula culminada por 5 dientes, hermafroditas, las externas del capítulo algo mayores que las internas. Corola vilosa en la base del limbo, amarilla, a menudo las más externas del capítulo con el dorso rosado o purpúreo, por lo general con los dientes de la lígula provistos de un mucrón subapical \pm desarrollado. Anteras amarillas. Ramas estilares amarillas. Aquenios homomorfos o dimorfos, los homomorfos con pico \pm desarrollado y con vilano, los dimorfos con los aquenios más externos del capítulo con pico escasamente diferenciado y sin vilano o con éste vestigial, y los internos con pico \pm de la longitud del cuerpo y con vilano, todos con 5 costillas longitudinales cubiertas de espículos escuamiformes dispuestos en filas transversales, \pm fusiformes, atenuados en los extremos del cuerpo, de un pardo-obscuro. Vilano blanco, caedizo, formado por 10(11) escamas setiformes, planas en la mitad inferior, plumosas, libres entre sí, dispuestas en 1 fila, por lo general también con otra fila externa muy pequeña de pelos escábridos. $x=6$.

\section{Observaciones}

Género monofilético que integran c. 8 especies distribuidas por la Región Mediterránea. Estudios con marcadores moleculares, nucleares y cloroplásticos, han mostrado que el género Achyrophorus es el hermano filogenético del género Hypochaeris.

\section{Clave para las especies}

1. Planta sufruticosa, glauca, glabra o glabrescente; involucro con 3 series de brácteas; brácteas internas del involucro sin setas en el nervio medio del dorso, o con setas dispuestas en 1 fila; aquenios homomorfos, todos con vilano 2. A. rutea

- Planta anual, verdosa, con indumento al menos en las hojas; involucro con 2 series de brácteas; brácteas internas del involucro con 2 filas de setas dispuestas a lo largo del nervio medio del dorso; aquenios dimorfos, los externos con pico \pm corto y sin vilano o éste vestigial, y los internos con pico bien diferenciado y con vilano... .. 2

2. Capítulos erectos antes de la antesis; brácteas internas del involucro con más de 2 filas de setas en la base del dorso; pedúnculo y brácteas del involucro por lo general con setas blanquecinas; dientes de las lígulas con un mucrón subapical de 0,1-0,2 $\mathrm{mm}$; aquenios de 0,2-0,4 $\mathrm{mm}$ de anchura 1. A. valdesii

- Capítulos péndulos antes de la antesis; brácteas internas del involucro solo con 2 filas de setas en el dorso; pedúnculo y brácteas del involucro 
con setas por lo general negruzcas; dientes de las lígulas sin mucrón subapical, o con un mucrón menor de $0,1 \mathrm{~mm}$; aquenios de 0,5-0,8 $\mathrm{mm}$ de anchura

3. A. stuessyi

1. Achyrophorus valdesii F.J. Jiménez, M. Ángeles \& M. Talavera, nom. nov.

$\equiv$ Hypochaeris achyrophorus L., Sp. Pl.: 810 (1753), sin. subst.

Ind. loc.: "Habitat in Creta.".

Lectotype: designado por Turland in Jarvis \& Turland (1998: 363; Herb. Clifford: 385, Hypochaeris 1: BM 000646854).

= Seriola aethnensis L., Sp. Pl., ed. 2, 2: 1139 (1763)

= Seriola depressa Viv., Fl. Cors. Prodr., App. 2: 4 (1830)

$\equiv$ Hypochaeris aethnensis subsp. depressa (Viv.) Arcang., Comp. Fl. Ital.: 415 (1882)

= Seriola caespitosa Porta in Nuovo Giorn.

Bot. Ital. 19: 310 (1887)

= Hypochaeris aethnensis subsp. foliosa

Arcang., Comp. Fl. Ital.: 415 (1882)

\section{Ecología, fenología y distribución}

Campos y cultivos, márgenes de caminos, pie de acantilados calcáreos, arenales costeros, etc.; 0-1300 m. (III)IV-V(VI). Sur de Europa, desde Francia hasta Rumanía, Grecia, Chipre, Turquía, N de África (N de Marruecos, Argelia, Túnez y Libia) e islas del Mediterráneo (Baleares, Córcega, Cerdeña, Sicilia y Creta). Islas Baleares. Esp.: PM[Mll Mn Ib Cabrera].

\section{Achyrophorus rutea (Talavera) Talavera, comb. nov.}

$\equiv$ Hypochaeris rutea Talavera in Lagascalia 9: 234 (1980) [basión.]

Holotype. SPAIN. Córdoba: Priego de Córdoba, Sierra Horconera, rocas calcáreas, VI.1960, Borja (SEV 5649; MAF 102406 \& 70913, ysotype).

= Hypochaeris leontodontoides var. glauca
N. Gallard \& Favarger in Candollea 40: 232 (1985)

Ecología, fenología y distribución

Grietas de acantilados calcáreos; 1000-1200 m. (V)VI. S de España y NW de Marruecos. Subbética cordobesa. Esp.: Co.

\section{Observaciones}

Achyrophorus rutea es muy parecida desde el punto de vista morfológico a Achyrophorus laevigatus (L.) Talavera \& M. Talavera comb. nov. [三 Seriola laevigata L., Sp. P1., ed. 2, 2: 1139 (1763), basión.; 三 Hypochaeris laevigata (L.) Ces. et al., Comp. Fl. Ital.: 465 (1878). Neotipo: designado por Turland in Jarvis \& Turland (1998: 366; BM000576303)], de Italia, Sicilia y Túnez de la que se diferencia sobre todo porque el vilano en $A$. laevigatus es biseriado, con una fila externa de pelos escábridos y otra interna de escamas plumosas, además de presentar por lo general hojas verdes y muy pelosas.

Del norte de África son también un puñado de especies relacionadas con esta y que se recogen seguidamente.

En el Atlas argelino, viviendo en grietas de roquedos calcáreos, aparece Achyrophorus decipiens (Cass.) Talavera, comb. nov. [三 Piptopogon decipiens Cass. in Cuvier, Dict. Sci. Nat. 48: 507 (1827), basión.; E Seriola laevigata Def., Fl. Atl. 2: 237, tab. 216 (1799), non L. (1763); 三 Piptopogon laevigatum Sch. Bip. in Nov. Act. Acad. Caes. Leopol.- Carol. Nat. Cur. 21: 165 (1845), nom. illeg.], una planta glabra con tallos ramificados. También de aquí y con una ecología similar es Achyrophorus hieracioides (Desf.) Talavera, comb. nov. [三 Seriola hieracioides Desf. in Méd. Eclairée Sci. Phys. 3:162 (1792), basión.], de hojas glabras, tallos afilos y monocéfalos, y aquenios sin pico.

En los acantilados marítimos del noroeste de Argelia vive Achyrophorus saldensis (Batt.) Talavera \& Tremetsberger, comb. nov. [三 Hypochaeris saldensis Batt. in Bull. Soc. 
Bot. France 59: 423 (1912), basión.; 三 Seriola saldensis Batt. in Bull. Soc. His. Nat. Africa N. 3: 23 (1912)], una planta muy singular, con cepa muy pelosa, algodonosa, rodeando a los tallos ramificados; las hojas son largamente pecioladas, espatuladas, laxamente hirsutas, enteras o algo dentadas, y los aquenios de c. $5 \mathrm{~mm}$, los externos sin pico y los centrales con un pico muy corto, hasta de $0,5 \mathrm{~mm}$, y el vilano de unos $8 \mathrm{~mm}$, formado por 5 escamas subuladas y plumosas. La especie fue descrita con material de Cap Noir [Lectotipo (designado aquí por M. Talavera). "Université d'Algerie / Herbier d'Afrique du Nord / Hypochoeris (Piptopogonopsis) saldensis Batt. / Type! / C. Bougie, falaises calcaires mariti / mes du Cap Noir / juin 1914 / Leg. Battandier [manuscrito Battandier]", etiqueta única (MPU-006482)] y que incluye cuatro especímenes, de los que se ha elegido el de la parte inferior izquierda como lectotipo; los otros tres, más tres plantas que se encuentran en el pliego MPU-006483, son isolectotipos.

En grietas de rocas del Atlas Medio y Gran Atlas de Marruecos, por encima de $2000 \mathrm{~m}$, vive Achyrophorus leontodontoides (Ball) Talavera \& Terrab, comb. nov. [三 Hypochaeris leontodontoides Ball in J. Bot. 11: 371 (1873), basión.]. Esta especie tiene el rizoma vertical, muy leñoso y ramificado, y las plantas por lo general no sobrepasan los $10 \mathrm{~cm}$ de altura; todas las hojas se disponen en la base, son enteras o débilmente dentadas, a veces casi seríceas, y los tallos escapiformes y monocéfalos; los capítulos tienen un involucro cubierto por un indumento aracnoideo, que falta en las demás especies del género. Es la especie basal del género, lo que han puesto en evidencia estudios de filogenia con marcadores nucleares y plastidiales (Tremetsberger et al., 2005).

3. Achyrophorus stuessyi F. J. Jiménez, M. Á. Ortiz \& M. Talavera, sp. nov.

- Hypochaeris achyrophorus auct., non L. (1753)
Holotype: SPAIN. Sevilla. Morón de la Frontera. Sierra Espartero, 14-IV-2013, F.J. Jiménez \& M.Á. Ortiz (SEV-286475). Isotype: ibídem (SEV -286476).

Paratype. SPAIN. Almería. Abrucena, 14-V-2011, M. Talavera \& S. Talavera (SEV-268807). Cádiz. Entre Ubrique y Grazalema, 13-VI-1970, E.F. Galiano \& B. Valdés (SEV-124208); San José del Valle, Sierra de las Cabras, 14-V-1976, M. J. Díez \& S. Silvestre (SEV-51423); Algodonales, Sierra de Líjar, 10-V-1980, A. Aparicio (SEV-60357); Entre Zahara de la Sierra y Puerto de Las Palomas, 4-V-1989, M. Arista \& S. Talavera (SEV-136639). Córdoba. Fuente Alhama, 14-V-1982, J. Arroyo (SEV-85726); Rute. Fuente El Chorrillo, 16-V-1980, M.J. Gallego, C. Romero \& S. Silvestre (SEV-54326). Granada. Alpujarra. Yegen, 21-5-1976, B. Cabezudo, S. Talavera, B. Valdés (215649). Puerto de la Ragua, 19-VI-1988, G. Alziar et al. (SEV-158974); Jerez del Marquesado, La Dehesa, 15-VI-1988, G. Alziar et al. (SEV-152631). Málaga. Cala del Moral, 19-IV-2011, J. A. Devesa (SEV-273730); Estepona, Sierra Bermeja, 16-V-1980, B. Molesworth (SEV-113089); Ronda. Monte de la Peineta, 19-IV-1974, S. Talavera \& B. Valdés (SEV-60858); Casa Bermeja, El Corte, alrededores del hotel El Corte, 21-IV-2013, D. Campos \& S. Talavera (SEV-282693). MOROCCO. Tetuán. Entre Mediq y Tetuán, a $1 \mathrm{~km}$ de Mediq hacia Cabo Negro, 16-V-2006, S. Talavera et al. (SEV-217531). Montañas del Rif. Aknoul, entre Kassita y Aknoul, Tizi Ouzli, 16-VI-2003, S. Talavera et al. (SEV-215534). Xaouen, entre Bab Taza y Bab Berred, 23-VI-1994, M. Arista, P. Gibbs, E. N. Lughadha, P.G. Murillo \& S. Talavera (SEV-149826). Atlas Medio. Tazzeka, entre SidiAbdallah-des-Rhiata y Bab-Azhar, 17-VI-2003, S. Talavera et al. (SEV-200413). Atlas Medio. Tizi ne Tiskine, 17-V-2006, S. Talavera et al. (SEV-218890). Montes de Zaïan, entre Tiddas y Jbel Bouchchene, 24-V-2006, S. Talavera et al. (277610)].

Plant annual like Achyrophorus valdesii from which it differs in having the capitula pendulous in pre-anthesis rather than erect; involucral bracts with two series of blackish spinulose setae of (1-) 3.5-4 mm on the median dorsal nerve, contrasting with the various series of whitish spinulose setae in A. valdesii; teeth of the ligules with a subapical mucron less than 
$0.1 \mathrm{~mm}$ or lacking a mucron; these are 0.1-0.2 mm in A. valdesii; achenes 0.5-0.7 mm broad, compared with 0.3-0.4 in A. valdesii.

Hierba anual, uni- o multicaule, pelosa en las hojas y generalmente también en la base de los tallos, con todos los pelos eglandulosos, unicelulares, blancos, con la base engrosada, provista también de un indumento farinoso muy laxo formado por pelos cortos y blanquecinos en el ápice de los pedúnculos y en la base del involucro; raíz axonomorfa, delgada. Tallos de de (8)20-40(50) cm x 1,5-3(4) $\mathrm{mm}$ de grosor (en la base), erectos, cilíndricos, estriados, escapiformes o foliosos, con 1-2(3) hojas bien desarrolladas, generalmente vilosos en la base, ramificados casi desde la base o en la mitad superior, rara vez simples, con pelos blancos de 0,5-2 mm. Hojas basales 5-13 $\mathrm{x}(1,7) 2-3,5(4) \mathrm{cm}$, formando una roseta, oblanceoladas, de enteras a dentado-lobadas, atenuadas en la base del limbo hacia un pecíolo corto, pelosas por ambas caras, casi hirsutas, con pelos blancos de 0,4-2 $\mathrm{mm}$, con la base muy bulbosa, los del envés más rígidos y algo menores que los del haz; las culinares 2-4,5 x 0,9-3 cm, elípticas o cuneiformes, cortamente pecioladas, con indumento semejante al de las basales. Capítulos péndulos antes de la antesis, pedunculados, por lo general agrupados en una inflorescencia laxa y paniculiforme; pedúnculos 5-14,5(20) cm, cilíndricos, con indumento laxo y farinoso en el ápice, junto al involucro, casi oculto por las setas espinulosas y negruzcas de 1-2 mm. Involucro 6-13(15) x (3)6-9(10) mm, cilíndrico, poco acrescente en la fructificación, con 2 series de brácteas adpresas, la externa mucho más pequeña que la interna; brácteas lanceoladas, ciliadas en el ápice, con quilla dorsal muy desarrollada por la que discurren 2 filas de setas espinulosas y negruzcas de (1)3,5-4 mm, sin otras setas fuera de la quilla; las externas 1,5-3,5 x 0,2-0,7 mm, casi enteramente herbáceas; las internas (5)8-12 x 1,7-2,5 mm, con el margen membranáceo.
Receptáculo plano en la fructificación, paleáceo, con las páleas interflorales de 12-14 x 1,4-1,6 $\mathrm{mm}$, linear-subuladas, membranáceas, con el nervio medio bien marcado, alcanzando o no la altura del vilano en la fructificación. Corola de las flores más externas del capítulo de 11,5-15 mm, amarilla, con el dorso del limbo rosado; tubo $(2,7) 3-5 \mathrm{~mm}$, glabro; limbo (6)8,5-10(12,5) mm, viloso en la base, con los dientes de la lígula sin o con mucrón subapical blanquecino menor de $0,1 \mathrm{~mm}$. Anteras (2,2)3-3,5(4) mm, amarillas. Ramas estilares (0,7)1-1,5 mm, amarillas. Aquenios dimorfos, con 5 costillas gruesas cubiertas de espículos escuamiformes dispuestos en filas transversales, pardo-obscuros, casi negros; los más externos del capítulo de 4-5,5 x 0,5-0,8 $\mathrm{mm}$, con pico grueso de $(0,5) 1-2 \mathrm{~mm}$, asidos al receptáculo, encerrados en las brácteas internas del involucro, no dispersables, sin vilano; los internos de 5-8,5 x 0,5-0,7 mm, con pico de 2-4(5) $\mathrm{mm}$, de longitud mayor o menor que la del cuerpo, dispersables, con vilano. Vilano (4)4,5-5,5 mm, banco, caedizo, formado por 10 escamas dispuestas en 1 fila, subuladas, libres entre sí, anchas, planas y glabras en el tercio inferior, finas y plumosas en los dos tercios superiores, rodeadas o no de una segunda fila de pelos escábridos de 0,2-0,5 mm. $2 n=12$.

\section{Ecología, fenología y distribución}

Grietas de rocas y base de acantilados calcáreos, taludes esquistosos o margosos, $\mathrm{y}$ en general herbazales cercanos a rocas; (70)400-1600 m. IV-VI(VII). Marruecos y S de España. Sistema Bético, desde la Subbética de Cádiz, Sevilla y Córdoba hasta la Penibética de Almería. Esp.: Al Ca Co Gr Ma Se.

\section{Observaciones}

Especie morfológicamente muy parecida a $A$. valdesii con la que a menudo ha sido confundida. Estudios de filogenia molecular, nuclear y plastidial, han mostrado que $A$. stuessyi está más cercana a $A$. leontodontoides 
que a las demás especies del género (Jiménez et $a l$, en preparación). Se trata de un caso claro de convergencia evolutiva posiblemente impuesta por el ciclo de vida anual dentro de un ambiente inestable como el clima mediterráneo.

AGRADECIMIENTOS. Agradecemos al editor de esta serie en Acta Botanica Malacitana por sus comentarios y correcciones, a los conservadores de los herbarios que nos enviaron los materiales que figuran en el texto, a Pilar Fernández-Piedra y al Dr. Fco. Javier Salgueiro por la búsqueda bibliográfica, y al Dr. Peter Gibbs por su ayuda de los textos en inglés.

\section{BIBLIOGRAFÍA}

ALAVI, S.A-1983-Hypochaeris. In: Jafri \& El-Cadi (eds.), Flora of Lybia 107: 347. Al-Faateh University. Tripoli

BURDET, H. M.,A. CHARPIN \& F. JACQUEMOUD -1983- Types nomenclaturaux des taxa ibériques décrits par Boissier ou Reuter. IV. Cistacées à Composées. Candollea 38: 751-802.

CANDOLLE, A. P. De-1838-Prodromus systematics naturalis regni vegetabilis. Vol. 7. Paris.

DEFILIPPS, R. A. -1976- Hypochoeris. In: Tutin, T.G, V.H. Heywood, N.A. Burges, D.M. Moore, D.H. Valentine, S.M. Walters \& D.A. Webb (eds.), Flora Europaea Vol 4: 308-310. Cambrigde University Press. Cambridge.

ENKE, N., B. GEMEINHOLZER \& C. ZIDORN -2012- Molecular and phytochemical systematics of the subtribe Hypochaeridinae (Asteraceae, Cichorieae). Org. Divers. Evol. 12: 1-16.

GREUTER, W., M. AGHABABIAN \& G. WAGENITZ -2005- Vaillant on Compositae: Systematic concepts and nomenclatural impact. Taxon 54: 149-174.

JARVIS, C. E., F. L. BARRIE, D. M. ALLAN \& J. L. RAVEAL -1993- A list of Linnaean generic names and their Types. Regn. Veget. 127: 1-100

JARVIS, C. E. \& N. J. TURLAND -1998Typification of Linnaean specific and varietal names in the Compositae (Asteraceae). Taxon 47: 347-370.

OBERPRIELER, M. A. \& R. VOGT -2002Hypochaeris arachnoidea Poir., a hiberto neglected species in NW Africa. Willdenowia 32: 231-136

ORTIZ, M. Á., S. TALAVERA, J.L. GARCÍACASTANO, K. TREMETSBERGER, T. STUESSY, F. BALAO \& R. CASIMIROSORIGUER -2006- Self-incompatibility and floral parameters in Hypochaeris sect. Hypochaeris (Asteraceae). Am. J. Bot. 93: 234-244.

ORTIZ, M. Á., K. TREMETSBERGER, S. TALAVERA, T.F STUESSY \& J.L. GARCÍA-CASTAÑO -2007- Population structure of Hypochaeris salzmanniana DC. (Asteraceae), an endemic species to the Atlantic coast on both sides of the Strait of Gibraltar, in relation to Quaternary sea level changes. Mol. Ecol. 16: 541-552.

ORTIZ,M.Á.,K.TREMETSBERGER,T.F.STUESSY, A. TERRAB, J. L. GARCÍA-CASTAÑO \& S. TALAVERA-2009- Phylogeographic patterns in Hypochaeris section Hypochaeris (Asteraceae, Lactuceae) of the western Mediterranean. $J$. Biogeogr. 36: 1384-1397.

PARKER, J. S. -1975- Aneuploidy and isolation in two Hypochoeris species. Chromosoma 52(1): 89-101.

TREMETSBERGER, K., S. TALAVERA, T. F. STUESSY, M. Á. ORTIZ, H. WEISSSCHNEEWEISS \& G. KADLEC -2004Relationship of Hypochaeris salzmanniana (Asteraceae, Lactuceae), an endangered species of the Iberian Peninsula, to $\mathrm{H}$. radicata and $\mathrm{H}$. glabra and biogeographical implications. Bot. J. Linn. Soc. 146: 79-95.

TREMET SB ER GER，K., H. WEIS S SCHNEEWEISS, T. STUESSY, R. SAMUEL, G. KADLEC, M. Á. ORTIZ \& S. TALAVERA -2005- Nuclear ribosomal DNA and karyotypes indicate a NW African origin of South American Hypochaeris (Asteraceae, Cichorieae). Mol. Phylogenet. Evol. 35: 102-116.

TREMETSBERGER, K., B. GEMEINHOLZER, H. ZETZSCHE, S. BLACKMORE, N. KILIAN \& S. TALAVERA -2013- Divergence time estimation in Cichorieae (Asteraceae) using a fossil-calibrated relaxed molecular clock. Org. Divers. Evol. 13: 1-13.

Dirección de los autores. Departamento de Biología Vegetal y Ecología (Botánica). Facultad de Biología, apartado 1095.41080-Sevilla.mtalavera@us.es 